\title{
Additive Manufacture of Dynamic Thiol-ene Networks Incorporating Anhydride-derived Reversible Thioester Links
}

Maciej Podgórskia,b, Sijia Huanga, Christopher N. Bowman*a

aDepartment of Chemical and Biological Engineering, University of Colorado, UCB 596, Boulder 80303 CO (USA)

${ }^{b}$ Department of Polymer Chemistry, Institute of Chemical Sciences, Faculty of Chemistry, Maria Curie-Sklodowska University, M. Curie-Sklodowska Sq. 5, 20-031 Lublin (Poland) *corresponding author e-mail: Christopher.Bowman@colorado.edu
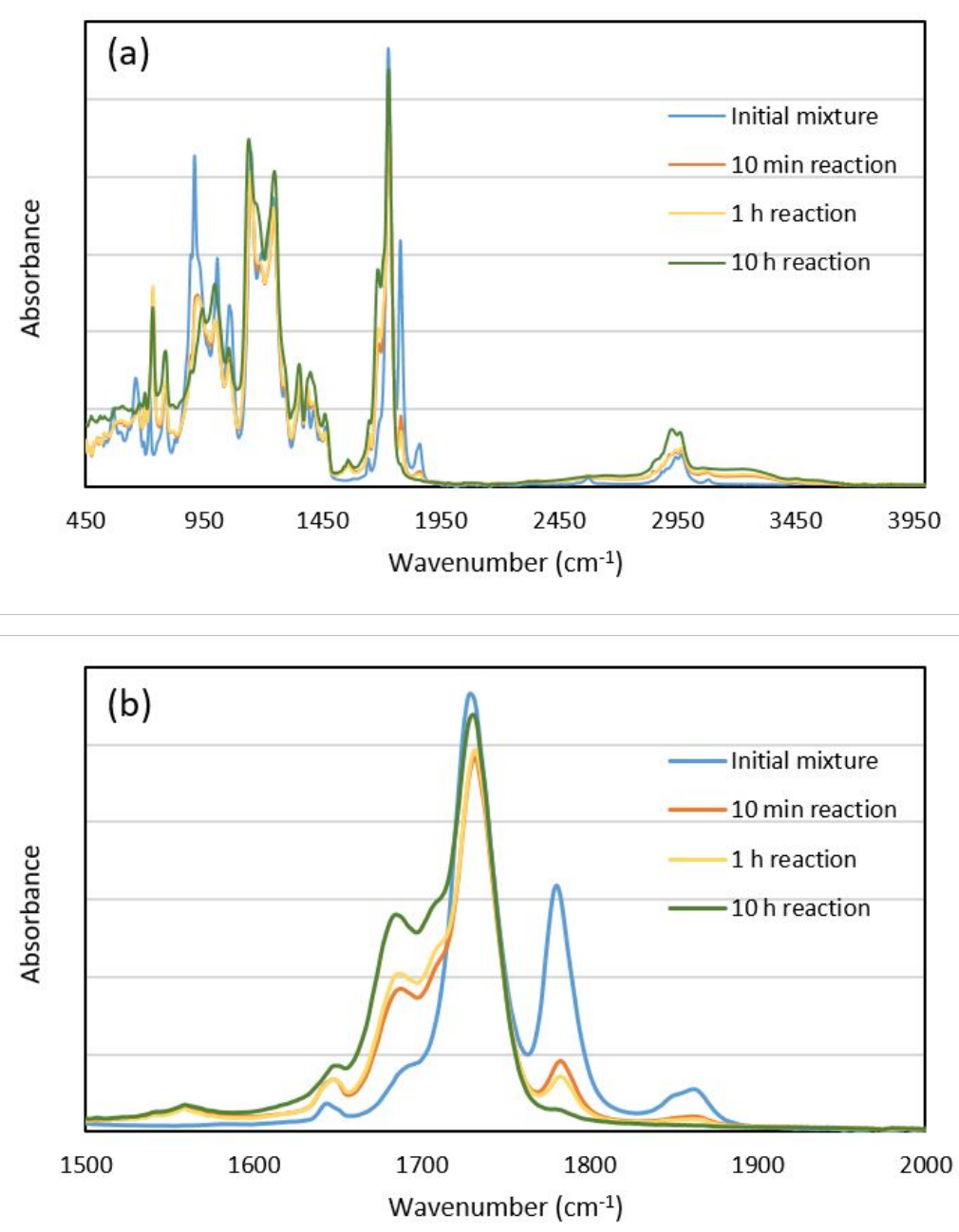

Figure S1. ATR-IR spectra for ASA/TMPTMP/DAC resin. (a) full mid-IR spectral range; (b) thioester and anhydride carbonyl and anhydride ring peak signals in the range of 1600-1900 $\mathrm{cm}^{-1}$. Evident is anhydride ring opening reaction resulting in $85 \%$ conversion after 1 hour at ambient temperature. 


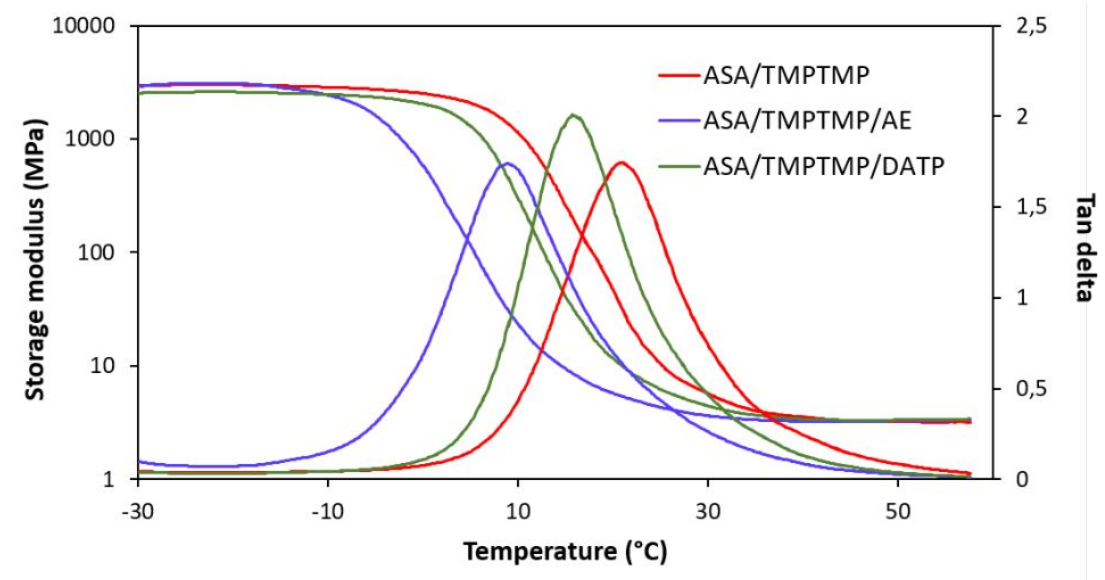

Figure S2. DMA data (storage moduli and tangent delta) for dynamic thiol-ene materials.
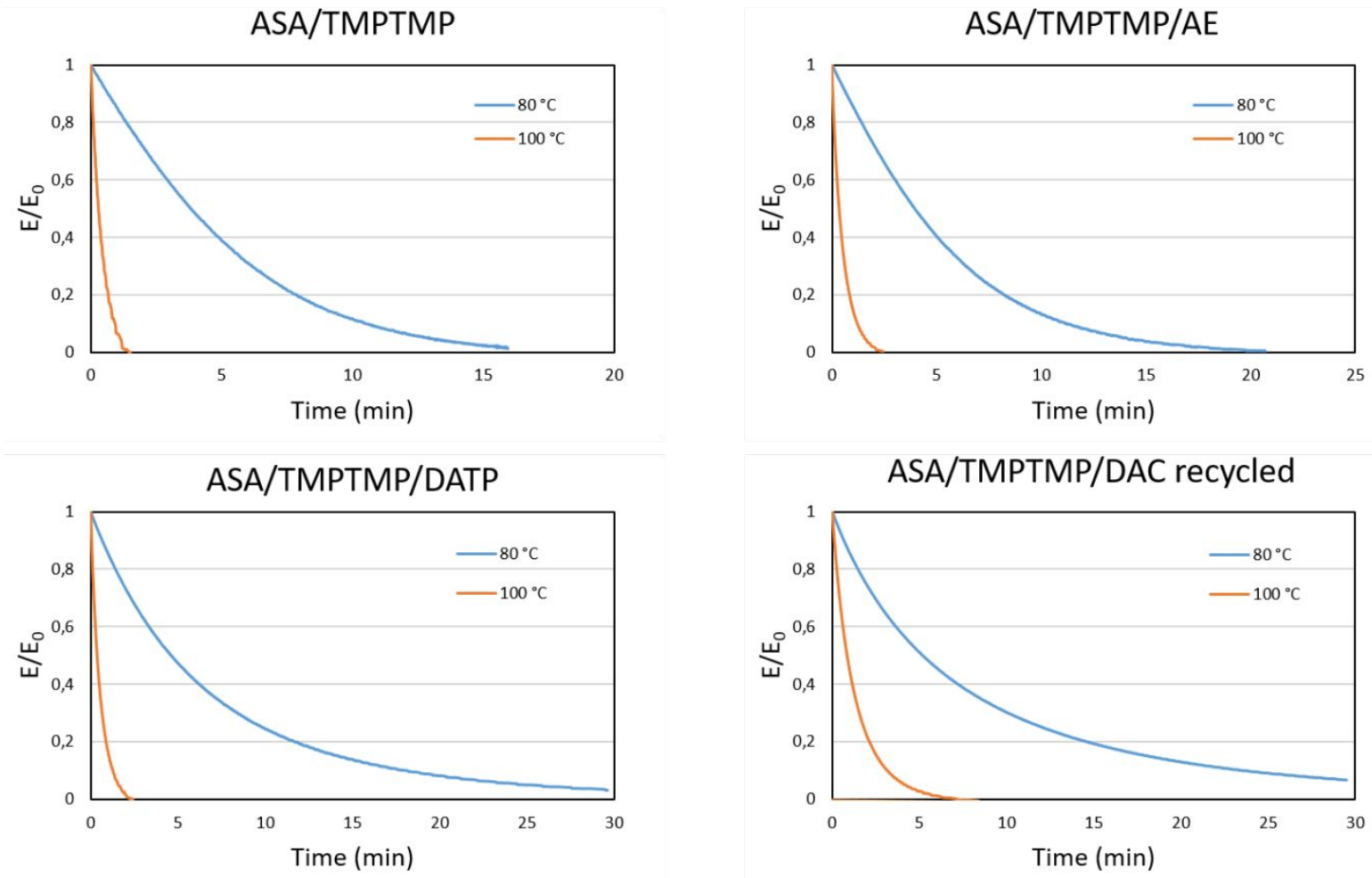

Figure S3. DMA stress relaxation data for dynamic thiol-ene materials collected at two temperatures. 


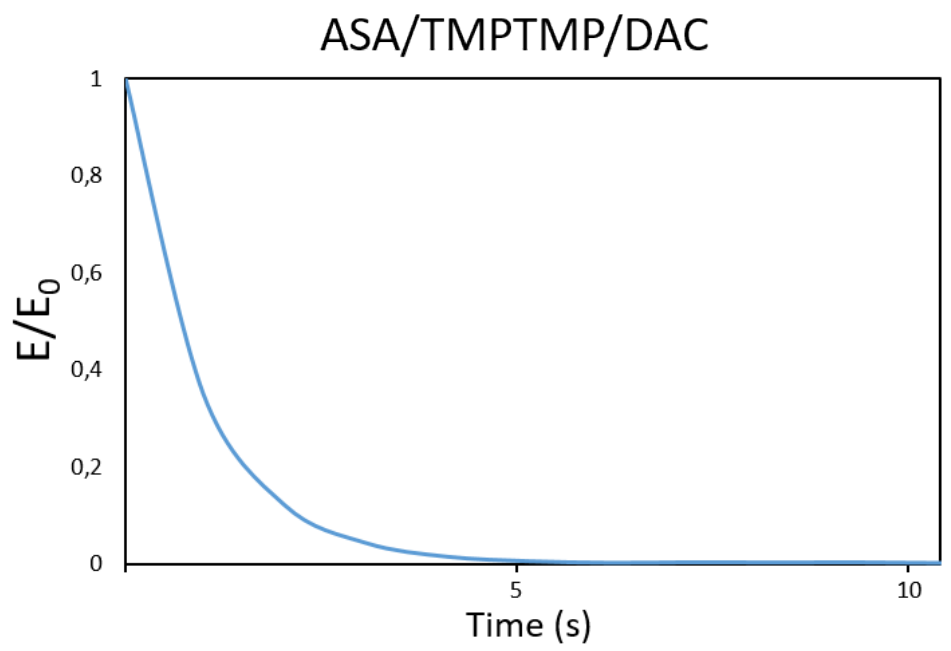

Figure S4. Stress relaxation at $140{ }^{\circ} \mathrm{C}$ for ASA/TMPTMP/DAC dynamic thiol-ene photopolymer.
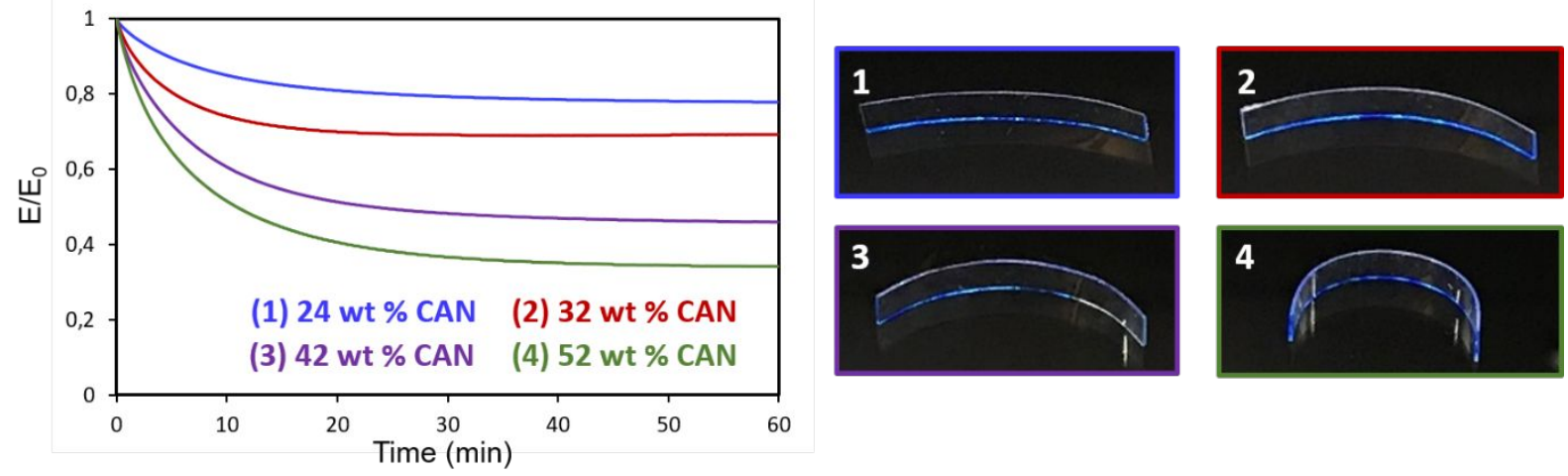

Figure S5. Stress relaxation at $120^{\circ} \mathrm{C}$ for semi-dynamic materials containing various loadings of recycled CAN resin. The nondynamic components were made of a stoichiometric mixture of HDT and TTT. Partial reshaping capabilities are also demonstrated in pictures 1 to 4 . 